\author{
A Simple Axiomatization of Nonadditive Expected Utility \\ Author(s): Rakesh Sarin and Peter Wakker \\ Source: Econometrica, Vol. 60, No. 6 (Nov., 1992), pp. 1255-1272 \\ Published by: The Econometric Society \\ Stable URL: http://www.jstor.org/stable/2951521 \\ Accessed: 04/05/2011 04:50
}

Your use of the JSTOR archive indicates your acceptance of JSTOR's Terms and Conditions of Use, available at http://www.jstor.org/page/info/about/policies/terms.jsp. JSTOR's Terms and Conditions of Use provides, in part, that unless you have obtained prior permission, you may not download an entire issue of a journal or multiple copies of articles, and you may use content in the JSTOR archive only for your personal, non-commercial use.

Please contact the publisher regarding any further use of this work. Publisher contact information may be obtained at http://www.jstor.org/action/showPublisher?publisherCode=econosoc.

Each copy of any part of a JSTOR transmission must contain the same copyright notice that appears on the screen or printed page of such transmission.

JSTOR is a not-for-profit service that helps scholars, researchers, and students discover, use, and build upon a wide range of content in a trusted digital archive. We use information technology and tools to increase productivity and facilitate new forms of scholarship. For more information about JSTOR, please contact support@jstor.org. 


\title{
A SIMPLE AXIOMATIZATION OF NONADDITIVE EXPECTED UTILITY
}

\author{
By Rakesh Sarin and Peter Wakker ${ }^{1}$
}

\begin{abstract}
This paper provides an extension of Savage's subjective expected utility theory for decisions under uncertainty. It includes in the set of events both unambiguous events for which probabilities are additive and ambiguous events for which probabilities are permitted to be nonadditive. The main axiom is cumulative dominance, which adapts stochastic dominance to decision making under uncertainty. We derive a Choquet expected utility representation and show that a modification of cumulative dominance leads to the classical expected utility representation. The relationship of our approach with that of Schmeidler, who uses a two-stage formulation to derive Choquet expected utility, is also explored. Our work may be viewed as a unification of Schmeidler (1989) and Gilboa (1987).
\end{abstract}

KEYwORDS: Ambiguity, nonadditive probability, stochastic dominance, rank-dependent utility, nonexpected utility.

\section{INTRODUCTION}

SAVAGE's (1954) SubJective EXPECTED utiLITy (SEU) theory has been widely adopted as the guide for rational decision making in the face of uncertainty. In SEU theory both the probabilities and the utilities are derived from preferences (see also Ramsey (1931)). This represents a hallmark contribution, as it avoids the reliance on introspection for quantifying tastes and beliefs. We continue in Savage's vein and extend his theory to derive a more general nonadditive expected utility representation, called Choquet expected utility (CEU). Schmeidler (1989, first version 1982) made the first contribution in providing a CEU representation and Gilboa (1987) extended this work. We develop this line of research further by providing an intuitive axiomatization of CEU.

The key distinction between our work and that of Savage is that we identify two types of events - unambiguous and ambiguous. People feel relatively "sure" about the probabilities of unambiguous events. An example of an unambiguous event could be the outcome of a toss of a fair coin (heads or tails). We assume that Savage's axioms hold for a sufficiently rich set of "unambiguous acts", i.e., acts measurable with respect to the unambiguous events. The probabilities of ambiguous events, however, are not known with precision. An example of such an event could be next week's weather conditions (rain or sunshine). Ambiguity in the probability of such events may be caused, for example, by a lack of available information relative to the amount of conceivable information (Keynes (1921)). Most people exhibit a reluctance to bet on events with ambiguous

\footnotetext{
${ }^{1}$ The support for this research was provided in part by the Decision, Risk, and Management Science branch of the National Science Foundation; the research of Peter Wakker has been made possible by a fellowship of the Royal Netherlands Academy of Arts and Sciences, and a fellowship of the Netherlands Organization for Scientific Research.

The authors are thankful to two referees for many detailed comments.
} 
probabilities. This reluctance leads to a violation of Savage's sure-thing principle (P2). The Choquet expected utility theory proposed here does not impose the sure-thing principle for all events and is therefore capable of permitting a liking for specificity and a dislike for ambiguity in probability.

The key condition in this paper to provide the Choquet expected utility representation is "cumulative dominance" (P4 in Section 3). Simply stated, this condition requires that if receiving consequence $\alpha$ or a superior consequence is considered more likely for an act $f$ than for an act $g$, for every $\alpha$, then the act $f$ is preferred to the act $g$. This condition is trivially satisfied for an SEU maximizer. Unlike the sure-thing principle that forces the probabilities for all events to be additive, cumulative dominance permits that probabilities for some events could be nonadditive. A probability function is nonadditive if the probability of the union of two disjoint events is not equal to the sum of the individual probabilities of each event. An example below will show how nonadditive probabilities could accommodate an aversion toward ambiguity.

The judgments and preferences that may lead to nonadditive probability have been rationalized by many authors. For example, Keynes (1921) has argued that confidence in probability influences decisions under uncertainty. Knight (1921) made the distinction between risk and uncertainty based on whether the event probabilities are known or unknown. Recently Schmeidler (1989) has argued that the amount of information available about an event may influence probabilities in such a way that probabilities are not necessarily additive.

In a seminal paper, Ellsberg (1961) showed that if one accepts Savage's definition of probability then a majority of subjects violates additivity of probability. Numerous experiments since then have confirmed Ellsberg's findings. Even though Ellsberg's example is well known we present it as it serves to illustrate the motivation and direction for our proposed modification of Savage's theory. Suppose an urn is filled with 90 balls, 30 of which are red $(R)$ and 60 of which are white $(W)$ and yellow $(Y)$ in an unknown proportion. One ball will be drawn randomly from the urn and your payoff will depend on the color of the drawn ball and the "act" (decision alternative) you choose. See Table I.

When subjects are asked to choose between acts $f$ and $g$, a majority chooses act $f$, presumably because in act $f$ the chance of winning $\$ 1000$ is precisely known to be $1 / 3$. In act $g$ the chance of drawing a white ball is ambiguous since the number of white balls is unknown. Now, when the same subjects are asked

TABLE I

The Ellsberg Options

\begin{tabular}{|c|c|c|c|}
\hline \multirow[b]{2}{*}{ Act } & \multirow{2}{*}{$\frac{30 \text { balls }}{\text { Red }}$} & \multicolumn{2}{|c|}{60 balls } \\
\hline & & White & Yellow \\
\hline$f$ & $\$ 1000$ & $\$ 0$ & $\$ 0$ \\
\hline$g$ & $\$ 0$ & $\$ 1000$ & $\$ 0$ \\
\hline$f^{\prime}$ & $\$ 1000$ & $\$ 0$ & $\$ 1000$ \\
\hline$g^{\prime}$ & $\$ 0$ & $\$ 1000$ & $\$ 1000$ \\
\hline
\end{tabular}


to choose between acts $f^{\prime}$ and $g^{\prime}$, a majority chooses the act $g^{\prime}$. Again, in act $g^{\prime}$, the chance of winning $\$ 1000$ is precisely known to be $2 / 3$, whereas in act $f^{\prime}$, the chance of winning is ambiguous. Thus, subjects tend to like specificity and to avoid ambiguity. By denoting $v(R), v(W)$, and $v(Y)$ as the probability of drawing a red, white, or yellow ball respectively, we obtain, assuming expected utility with $u(0)=0: f \succ \mathrm{g}$ implies

$$
v(R) u(1000)>v(W) u(1000), \quad \text { or } \quad v(R)>v(W) ;
$$

$g^{\prime} \succ f^{\prime}$ implies

$$
\begin{aligned}
& v(W) u(1000)+v(Y) u(1000)>v(R) u(1000)+v(Y) u(1000), \\
& v(W)>v(R) .
\end{aligned}
$$

Thus, consistent probabilities cannot be assigned to the states, as $v(R)$ cannot simultaneously be larger as well as smaller than $v(W)$. Clearly, in the above example no inconsistency results if $v(R \cup Y) \neq v(R)+v(Y)$. In our development we permit nonadditive probabilities for some events (such as $R \cup Y$ ) that we call ambiguous events. Our strategy is to differentiate between ambiguous and unambiguous events by requiring that only the acts that are measurable with respect to unambiguous events satisfy Savage's axioms. General acts are assumed to satisfy somewhat weaker conditions that may yield nonadditive probabilities for ambiguous events. It is to be noted that we do not require an a priori definition of unambiguous or ambiguous events (for the latter see Fishburn (1991)). We do, however, assume that there exists a subclass of events, such as those generated by a roulette wheel, such that an SEU representation holds with respect to these events. The idea is that these events are unambiguous. The subclass of unambiguous events should be rich enough to ensure that all ambiguous events can be calibrated by appropriate bets contingent on unambiguous events.

The strategy of permitting probabilities to be nonadditive and using them in CEU was first proposed by Schmeidler (1989, first version 1982). Schmeidler uses the set-up of Anscombe \& Aumann (1963) (as refined in Fishburn (1967, $1970,1982)$ ), where for every state an act leads to an objective probability distribution, to formulate his axioms and derive the result. A nonadditive probability extension for the approach of Savage (1954) in full generality is very complicated. Gilboa (1987) succeeded in finding such an extension. The resulting axioms are, however, quite complicated and do not seem to have simple intuitive interpretations (see Fishburn (1988, page 202)). In this paper, we propose another extension of Schmeidler's model that in our view has a greater intuitive appeal. The basic idea is to reformulate Savage's axioms to permit nonadditivity in probability for ambiguous events (event $R \cup Y$ in Table I) while preserving additivity for unambiguous events (event $Y \cup W$ in Table I). Technically, our work may be viewed as a sort of unification of Gilboa (1987) and Schmeidler (1989), and builds heavily on these works. Additional axiomatizations of CEU that assume some rich structure on the consequences instead of the states have been provided in Wakker (1989a, b, 1990a), and Nakamura 
(1990, 1992). Wakker (1990b) has shown that CEU when applied to decision making under risk (where probabilities are extraneously specified) is identical to rank-dependent (anticipated) utility. A survey of several independent discoveries of the CEU form has been given in Wakker (1991a).

Schmeidler's lottery-acts formulation may be viewed as a two-stage process where a state $s$ occurs in the first stage and in the second stage a lottery is played to determine the final consequence. If probabilities are additive the one-stage formulation (e.g., of Savage) and the two-stage formulation (e.g., of Anscombe and Aumann) yield the same conclusion. However, as we shall see, in the nonadditive case the two formulations yield different conclusions about the preference rankings of acts.

We begin by presenting some notations and definitions in Section 2. Our axioms and main result are stated in Section 3. In Section 4 we explore the relationship between CEU and SEU models. An example and a general result showing the irreconcilability of Schmeidler's two-stage formulation with a naturally equivalent one-stage formulation are presented in Section 5. Finally, conclusions are contained in Section 6, and proofs are given in the Appendix.

\section{DEFINITIONS}

\subsection{Elementary Definitions}

In this section we present the notation for the Savage (1954) style formulation for decisions under uncertainty and introduce some definitions that are useful in developing our results. There is a set $b$ of consequences (payoffs, prizes, outcomes) and a set $S$ of states of nature. The states in $S$ are mutually exclusive and collectively exhaustive, so that exactly one state is the true state. We shall let $\mathscr{A}$ denote a $\sigma$-algebra of subsets of $S$, i.e., $\mathscr{A}$ contains $S, A \in \mathscr{A}$ implies $A^{c}$ (the complement of $A$ ) $\in \mathscr{A}$, and $\mathscr{A}$ is closed under countable unions (this will be generalized in Remark 3.3). Thus $\mathscr{A}$ also contains $\varnothing$, and is closed under countable intersections. Subjective probabilities or "capacities" will be assigned to the elements of $\mathscr{A}$; these elements are called events. An event $A$ is informally said to occur if $A$ contains the true state. The set $b$ is also assumed to be endowed with a $\sigma$-algebra $\mathscr{D}$; this will only play a role for acts with an infinite number of consequences.

A decision alternative or an act is a function from $S$ to $b$ that is measurable, i.e., $f^{-1}(D) \in \mathscr{A}$ for all $D \in \mathscr{D}$. If the decision maker chooses an act $f$, then the consequence $f(s)$ will result where $s$ is the true state. The decision maker is uncertain about which state is true, hence about which consequence will result from an act. The set of acts is denoted as $\mathscr{F}$. Act $f$ is constant if, for some $\alpha \in \mathscr{b}, f(s)=\alpha$ for all states $s$. Often a constant act is identified with the resulting consequence. Statements of conditions are simplified by defining $f_{A}$ as the restriction of $f$ to $A, f_{A} h$ as the act that assigns consequences $f(s)$ to all $s \in A$, and consequences $h(s)$ to all $s \in S \backslash A$. Given that consequences are identified with constant acts, $f_{A} \alpha$ designates the act that is identical to $f$ on $A$ and constant $\alpha$ on $S \backslash A ; \alpha_{A} \beta$ is similar. Further, for a partition $\left\{A_{1}, \ldots, A_{m}\right\}$, 
we denote by $\alpha_{A_{1}}^{1} \ldots \alpha_{A_{m}}^{m}$ the act that assigns consequence $\alpha^{j}$ to each $s \in A_{j}$, $j=1, \ldots, m$. Such acts are called step acts. ${ }^{2}$ A binary relation $\geqslant$ over $\mathscr{F}$ gives the decision maker's preferences. The notations $\succ, \preccurlyeq, \prec$, and $\sim$ are as usual. Further, $\geqslant$ is a weak order if it is complete $(f \geqslant g$ or $g \geqslant f$ for all $f, g)$ and transitive.

We define $\geqslant$ on $\mathscr{b}$ from $\geqslant$ on $\mathscr{F}$ through constant acts: $\alpha \geqslant \beta$ if $f \geqslant g$ where $f$ is constant $\alpha, g$ is constant $\beta$. Postulate P3 will ensure that $\succcurlyeq$ on $\mathscr{F}$ and $\succcurlyeq$ on $\mathscr{b}$ are in proper agreement. We assume that $\succcurlyeq$ and $\mathscr{D}$ are compatible in the sense that all "preference intervals" are contained in $\mathscr{D}$. A preference interval, as defined in Fishburn (1982), is a set $E \subset D$ such that $\alpha, \gamma \in E, \alpha \geqslant \beta \geqslant \gamma$ imply $\beta \in E$. A special case is a set $E$ such that $\alpha \in E$, $\beta \succcurlyeq \alpha$ implies $\beta \in E$. Such sets are called cumulative consequence sets. They will play a central role in this paper. Example A.4 below shows why, in the absence of set continuity, cumulative dominance must include all cumulative consequence sets and not just sets of the form $\{\beta: \beta \succcurlyeq \alpha\}$; in the latter case cumulative dominance would become too strong.

Following Savage (1954) (see also de Finetti (1931, 1937) and Ramsey (1931)), we define $\succcurlyeq$ on $\mathscr{A}$ from $\succcurlyeq$ on $\mathscr{F}$ through "bets on events:" $A \geqslant B$ if there exist consequences $\alpha \succ \beta$ such that $\alpha_{A} \beta \geqslant \alpha_{B} \beta$. We then say that $A$ is more likely than $B$. Postulate P4 will ensure that $\succcurlyeq$ on $\mathscr{A}$ satisfies usual conditions such as transitivity and completeness, and is in proper agreement with $\geqslant$ on $\mathscr{F}$; see also Lemma 2.1 in Section 2.2. Obviously, in this paper the more-likelythan relation will not correspond to an additive probability; it will correspond to a "capacity", i.e. a nonadditive probability; see Lemma $2.1{ }^{3}$ We will make use of a sub $\sigma$-algebra $\mathscr{A}^{u a}$ of $\mathscr{A}$ that should be thought of as containing unambiguous events, for example events generated by the spin of a roulette wheel, or by repeated tosses of a coin. We denote by $\mathscr{F}$ ua the set of acts that are $\mathscr{D}-\mathscr{A}^{u a}$ measurable; i.e., $\mathscr{F}$ ua contains the acts $f$ for which $f^{-1}(E) \in \mathscr{A}^{\text {ua }}$ for each $E \in \mathscr{D}$. We will assume that Savage's (1954) axioms are satisfied if attention is restricted to the unambiguous events and $\mathscr{F} u$.

An event $A \in \mathscr{A}^{u a}$ is null if $f_{A} h \sim g_{A} h$ for all $f, g \in \mathscr{F}^{u a}$; it is non-null otherwise.

\subsection{Choquet Expected Utility}

A function $v: \mathscr{A} \rightarrow[0,1]$ is a capacity if $v(\varnothing)=0, v(S)=1$, and $v$ is monotonic with respect to set-inclusion, i.e., $A \supset B \Rightarrow v(A) \geqslant v(B)$. The capacity $v$ is a

\footnotetext{
${ }^{2}$ Every step act is "simple," i.e., is measurable and has a finite range. If $\mathscr{D}$ contains every one-element subset, then every simple act is a step act. Step acts turn out to be easier to work with than simple acts.

${ }^{3}$ Sometimes a nonadditive capacity is a strictly increasing transform of a probability measure, which then also represents the "more likely than" relation. In general, however, a capacity will not be of that form.
} 
( finitely additive) probability measure if, in addition, $v$ is additive, i.e.,

$$
v(A \cup B)=v(A)+v(B)
$$

for all disjoint $A, B$. A capacity $v$ is convex-ranged if for every $A \supset C$ and every $\mu$ between $v(A)$ and $v(C)$ there exists $A \supset B \supset C$ such that $v(B)=\mu$.

For a capacity $v$, and a measurable function $\phi: S \rightarrow \mathbb{R}$, the Choquet integral of $\phi$ (with respect to $v$ ), denoted $\int_{S} \phi d v$, or $\int \phi d v$, or $\int \phi$, and introduced in Choquet (1953-1954), is

$$
\int_{\mathbb{R}^{+}} v(\{s \in S: \phi(s) \geqslant \tau\}) d \tau+\int_{\mathbb{R}^{-}}[v(\{s \in S: \phi(s) \geqslant \tau\})-1] d \tau .
$$

In Wakker (1989b, Chapter VI) illustrations are given for the Choquet integral. We say that $\geqslant$ maximizes Choquet expected utility (CEU) if there exist a capacity $v$ on $\mathscr{A}$ and a measurable utility function $U: b \rightarrow \mathbb{R}$ such that the preference relation $\succcurlyeq$ is represented by $f \mapsto \int_{S} U(f(s)) d v$; the latter is called the Choquet expected utility of $f$, denoted $\operatorname{CEU}(f)$. Suppose there are $n$ states $s_{1}, \ldots, s_{n}$ and $U\left(f\left(s_{1}\right)\right) \geqslant \ldots \geqslant U\left(f\left(s_{n}\right)\right)$. Then

$$
\operatorname{CEU}(f)=\sum_{i=1}^{n-1}\left(U\left(f\left(s_{i}\right)\right)-U\left(f\left(s_{i+1}\right)\right)\right) v\left(\left\{s_{1}, \ldots, s_{i}\right\}\right)+U\left(f\left(s_{n}\right)\right) .
$$

The proof of the following lemma is left to the reader.

Lemma 2.1: If $\succcurlyeq$ on $\mathscr{F}$ maximizes $C E U$, then the relation $\succcurlyeq$ on $b$ is represented by the utility function $U$, and the relation $\geqslant$ on $\mathscr{A}$ is represented by the capacity $v$ whenever $U$ is nonconstant.

\section{THE MAIN RESULT}

Apart from the well-known postulates of Savage on the unambiguous acts, we shall use one additional postulate, "cumulative dominance" (P4 on the next page), to govern preferences over ambiguous acts. It is a natural extension of Savage's P4 to acts with more than two consequences. When restricted to acts with exactly two consequences, our P4 is identical to Savage's P4. It is best appreciated as an adaptation of the stochastic dominance condition. Let us recall that stochastic dominance applies to decision making under risk, where for each uncertain event $A \in \mathscr{A}$ a probability $P(A)$ is well specified, and usually $b$ is an interval within $\mathbb{R}$. In this setting, an act (or its probability distribution as generated over consequences) stochastically dominates another if it assigns to each cumulative consequence set ${ }^{4}$ at least as high a probability. In the present set-up, without probabilities attached to each event, it is natural to say that an act $f$ stochastically ("cumulatively") dominates an act $g$ if the decision maker regards each cumulative consequence set at least as likely under $f$ as under $g$. Monotonicity with respect to stochastic dominance, reformulated

\footnotetext{
${ }^{4}$ E.g., receiving $\alpha$ or a superior consequence.
} 
with this adaptation, is our additional postulate P4 below. It turns out that this condition in the presence of the usual conditions, and Savage's conditions on a rich set of unambiguous acts, is necessary and sufficient for CEU. To readers familiar with CEU and with Savage's set-up, the proof of the main result may be transparent if $\mathrm{P} 4$ is assumed. We hope that this mathematical simplicity is viewed as a strength of the paper, because P4, in our opinion, is an intuitively appealing assumption about behavior under uncertainty as well.

We first state the axioms and then the main theorem, which is followed by a discussion.

Postulate P1: Weak ordering.

Postulate P2 (The Sure-thing Principle for Unambiguous Acts): For all events $A$ and acts $f, g, h, h^{\prime}$ with $f_{A} h, g_{A} h, f_{A} h^{\prime}, g_{A} h^{\prime} \in \mathscr{F}$ ua:

$$
f_{A} h \geqslant g_{A} h \Leftrightarrow f_{A} h^{\prime} \succcurlyeq g_{A} h^{\prime} \text {. }
$$

Postulate P3: For all events $A \in \mathscr{A}$, acts $f \in \mathscr{F}$, and consequences $\alpha, \beta$ : $\alpha \succcurlyeq \beta \Rightarrow \alpha_{A} f \succcurlyeq \beta_{A} f$. The reversed implication holds as well if $A \in \mathscr{A}^{\text {ua }}, A$ is nonnull, and $f \in \mathscr{F}$ ua.

Postulate P4 (Cumulative Dominance): For all acts $f, g$ we have:

$$
f \succcurlyeq g \text { whenever } f^{-1}(E) \succcurlyeq g^{-1}(E)
$$

for all cumulative consequence sets $E$.

Postulate P5 (Nontriviality): There exist consequences $\alpha, \beta$ such that $\alpha \succ \beta$.

Postulate P6 (Fineness of the Unambiguous Events): If $\alpha \in b$ and, for $f \in \mathscr{F} u a, g \in \mathscr{F}, f \succ g$, then there exists a partition $\left(A_{1}, \ldots, A_{m}\right)$ of $S$, with all elements in $\mathscr{A}^{u a}$, such that $\alpha A_{j} f \succ g$ for all $j$, and the same holds with $\prec$ instead of $\succ$.

The following postulate is Gilboa's adaptation of Savage's P7 to the case of CEU. It is a technical condition, and is only needed for the extension of CEU to acts with infinite range. In order to state the postulate, we define an event $A$ to be $f$-convex if for any $s, s^{\prime \prime} \in A$ and $s^{\prime} \in S, f(s) \succ f\left(s^{\prime}\right) \succ f\left(s^{\prime \prime}\right) \Rightarrow s^{\prime} \in A$. Note below that, for some fixed $s \in A, f(s)_{A} h$ denotes the act that assigns $f(s)$ to each $s^{\prime} \in A$, and is identical to $h$ on $A^{c}$.

Postulate P7: For all $f, g \in \mathscr{F}$, and nonempty f-convex events $A$,

$$
f(s)_{A} f \geqslant g \text { for all } s \in A \Rightarrow f \succcurlyeq g,
$$

and the same holds with $\preccurlyeq$ instead of $\succcurlyeq$.

We now state the main theorem. In it, cardinal abbreviates "unique up to scale and location." 
THEOREM 3.1: The following two statements are equivalent:

(i) The preference relation $\geqslant$ maximizes CEU for a bounded nonconstant utility function $U$ on $\mathscr{b}$, and for a capacity $v$ on $\mathscr{A}$. On $\mathscr{A}^{\text {ua }}$ the capacity is additive and convex-ranged.

(ii) Postulates P1-P7 are satisfied.

Further, the utility function in statement $(i)$ is cardinal, and the capacity is unique.

In the above result condition P4 can be weakened to the following "cumulative reduction" condition, if in addition we include Savage's P4 (i.e., our P4 restricted to two-consequence acts). Cumulative reduction says that the only relevant aspect of an act is its "decumulative" distribution. Cumulative reduction follows from two-fold application of $\mathrm{P} 4$, with the roles of $f$ and $g$ interchanged. This condition is the only implication of P4 that we shall use in the proof of Theorem 3.1 for acts with more than two consequences. We have preferred to present the stronger P4 in the theorem because of its close relationship with stochastic dominance.

Postulate P4' (Cumulative Reduction): For all acts $f, g$ we have:

$$
f \sim g \text { whenever } f^{-1}(E) \sim g^{-1}(E)
$$

for all cumulative consequence sets $E$.

Let us also point out that all conditions can be weakened to hold only for step acts, with the exception of $\mathrm{P} 1$, the act $g$ in $\mathrm{P} 6$, and $\mathrm{P} 7$. If $\mathrm{P} 4 / \mathrm{P} 4^{\prime}$ is restricted to step acts then cumulative consequence sets can be restricted to sets of the form $\{\beta \in \mathscr{b}: \beta \succcurlyeq \alpha\}$ for some $\alpha \in \mathscr{b}$. The next example considers the cases where the state space is a product space. These are the cases considered by Schmeidler. The above theorem applies to any case where there is a sub $\sigma$-algebra isomorphic to the Borel sets on $[0,1]$ endowed with the Lebesgue measure; the latter is somewhat more general than product spaces. The technique of this paper allows for more generality: the sets of ambiguous acts and events can be quite general, as long as the set of unambiguous acts and events is sufficiently rich. This will be explicated in Remark 3.3. A further generalization can be obtained in our one-stage approach by imposing on $\mathscr{F}^{u a}$ the conditions of Gilboa (1987) which lead to CEU, instead of using Savage's conditions which lead to additive expected utility. The proof of this more general result is almost identical to the proof of Theorem 3.1. In other words, as soon as there is a sufficiently rich subset of acts on which CEU holds, then by cumulative dominance CEU will spread over all acts. Alternatively, for the rich subset of acts, we could have taken the set of probability distributions over the consequences, with expected utility or rank-dependent utility maximized there. We chose Savage's set-up because it is very appealing.

EXAMPLE 3.2: Let $[0,1]$ be endowed with the usual Lebesgue measure (i.e., uniform distribution) over the usual Borel $\sigma$-algebra. $\Omega$ can be any set endowed 
with any $\sigma$-algebra. Let $S=\Omega \times[0,1]$, endowed with the usual product $\sigma$-algebra; $v$ is any capacity that assigns the Lebesgue measure of $E$ to any set $\Omega \times E$. $b$ can be any arbitrary set, and $U: b \rightarrow \mathbb{R}$ any function, nonconstant to avoid triviality. Preferences maximize CEU. With $\mathscr{A}^{\text {ua }}$ the $\sigma$-algebra of all sets of the form $\Omega \times E$ for $E$ a Borel-subset of [0,1], all Postulates P1-P7 are satisfied.

Remark 3.3: The requirement that $\mathscr{A}$ should be a $\sigma$-algebra, and that all $\mathscr{A}$ - $\mathscr{D}$ measurable functions from $S$ to $b$ should be included in $\mathscr{F}$, can be restricted to the unambiguous acts and events, as follows.

(i) $\mathscr{A}^{u a}$ should be a $\sigma$-algebra, and all $\mathscr{A}^{u a}-\mathscr{D}$ measurable functions from $S$ to $b$ should be included in $\mathscr{F}$.

Then, in addition, the following adaptations should be made. First, the measurability requirement should be imposed that for all $f \in \mathscr{F}$ and cumulative consequence sets $E, f^{-1}(E) \in \mathscr{A}$. Second, Postulate $\mathrm{P} 3$ should be required only if $\alpha_{A} f, \beta_{A} f \in \mathscr{F}$. Third, the nontriviality Postulate P5 should be changed as follows:

Postulate P5': There exist consequences $\alpha \succ \beta$ such that $\alpha_{A} \beta_{A^{c}} \in \mathscr{F}$ for all events $A \in \mathscr{A}$.

$\mathrm{P5}^{\prime}$ as such is not a necessary condition for the CEU representation. Fourth and finally, for Postulate P7, needed for nonsimple acts, it should be required that for all acts $f \in \mathscr{F}, f$-convex events $A$, and states $s \in A, f(s)_{A} f$ be contained in $\mathscr{F}$ (consequences can be "collapsed").

Note that this allows for great generality. For instance, $\mathscr{A}$ may consist of $\mathscr{A}^{u a}$, events described by a roulette wheel, and a collection of events entirely unrelated to the roulette wheel. There is no need to incorporate intersections or unions of events described by the roulette wheel, and other events.

Let us finally comment further on the uniqueness of the capacity in Theorem 3.1. Suppose Statement (i) in Theorem 3.1 holds. Would there exist CEU representations that also represent the preference relation but have $v$ nonadditive on $\mathscr{A}^{\text {ua }}$ ? The following observation answers this question.

Observation 3.4: Suppose Statement (i) in Theorem 3.1 holds. If there exist three or more equivalence classes of consequences, then for any CEU representation the capacity will be additive on $\mathscr{A}^{u a}$. If there exist no more than two equivalence classes of consequences, then any capacity can be taken that is a strictly increasing transform of the capacity of Theorem 3.1.

${ }^{5}$ Only one will be additive on $\mathscr{A}^{u a}$ of course. 


\section{REVEALED UNAMBIGUOUS EVENTS}

In this section we characterize revealed unambiguous events and partitions, i.e., those for which the capacity is additive (defined below). It is possible that a decision maker considers some events as ambiguous but nevertheless reveals an additive capacity with respect to these. The characterization of this section will lead to a generalization of the theorem of Anscombe and Aumann (1963). A capacity is additive on a partition $\left\{A_{1}, \ldots, A_{m}\right\}$ if $v(A \cup B)=v(A)+v(B)$ for all disjoint events $A, B$ that are unions of elements of the partition. This is equivalent to additivity of the capacity on the algebra generated by the partition. A capacity is additive with respect to an event $A$ if it is additive with respect to the partition $\left\{A, A^{c}\right\}$, i.e., if $v(A)=1-v\left(A^{c}\right)$. Gilboa (1989) used the term symmetry for a capacity that is additive with respect to each event. As shown there, symmetry does not imply that the capacity is additive. A capacity is additive if and only if it is additive on each partition, which holds if and only if it is additive on each partition consisting of three events (consider, for disjoint events $A, B$, the partition $\left.\left\{A, B,(A \cup B)^{c}\right\}\right)$. In the presence of the rich $\mathscr{A}^{\text {ua }}$ in Theorem 3.1, the characterization of revealed unambiguous partitions is easy. Note that in CEU additivity of the capacity immediately leads to SEU. Machina and Schmeidler (1990) consider the case with an additive probability measure on the events, and a general (nonexpected utility) functional, such as used in Machina (1982). Like our main result, their main result weakens Savage's sure-thing principle and strengthens his P4. Their P4 implies the sure-thing principle for two-consequence acts, which our $\mathrm{P} 4$ obviously does not. In addition, it implies, mainly in the presence of P6, our P4. The Ellsberg paradoxes give examples where their $\mathrm{P} 4$ is violated while our P4 is satisfied.

Proposition 4.1: Suppose Statement (i) in Theorem 3.1 holds. Let $\left\{A_{1}, \ldots, A_{m}\right\}$ be a partition. The following four statements are equivalent:

(i) The capacity is additive on the partition.

(ii) For all disjoint $A$ and $A^{\prime}$ that are unions of elements of the partition, and for disjoint unambiguous events $B^{u a} \sim A, B^{u a^{\prime}} \sim A^{\prime}$, we have $A \cup A^{\prime} \sim B^{u a} \cup B^{u a^{\prime}}$.

(iii) There exists an unambiguous partition $\left\{B_{1}^{u a}, \ldots, B_{m}^{u a}\right\}$ such that

$$
\alpha_{A_{1}}^{1} \ldots \alpha_{A_{m}}^{m} \sim \alpha_{B_{1}^{u a}}^{1} \ldots \alpha_{B_{m}^{u a}}^{m} \quad \text { for all consequences } \alpha^{1}, \ldots, \alpha^{m} .
$$

(iv) For each unambiguous partition $\left\{B_{1}^{u a}, \ldots, B_{m}^{u a}\right\}$ we have:

$$
\begin{aligned}
& A_{1} \cup \cdots \cup A_{j} \sim B_{1}^{u a} \cup \cdots \cup B_{j}^{u a} \\
& \text { for all } j \Rightarrow \alpha_{A_{1}}^{1} \ldots \alpha_{A_{m}}^{m} \sim \alpha_{B_{1}^{u a}}^{1} \ldots \alpha_{B_{m}^{u a}}^{m} \quad \text { for all consequences } \alpha^{1}, \ldots, \alpha^{m} .
\end{aligned}
$$

We could obviously obtain additivity of the capacity $v$ in Statement (i) of Theorem 3.1 by adding any of the conditions in Statements (ii), (iii), or (iv) above, for each partition, to Statement (ii) of Theorem 3.1. Given the impor- 
tance of the result that can be derived from Statement (iv), let us make the condition explicit:

Postulate P4" (Reduction): For each partition $\left\{A_{1}, \ldots, A_{m}\right\}$ and each unambiguous partition $\left\{B_{1}^{u a}, \ldots, B_{m}^{u a}\right\}$, (2) holds true.

If in the definition of reduction we would have added the condition that the consequences in (2) are rank-ordered, i.e., $\alpha_{1} \geqslant \cdots \geqslant \alpha_{m}$, then the condition would have been identical to $\mathrm{P}^{\prime}$ (cumulative reduction) restricted to step acts, which is all of $\mathrm{P} 4$ that is needed apart from its restriction to two-consequence acts (i.e., Savage's P4). P4" resembles the reduction principle in Fishburn (1988), which is called neutrality in Yaari (1987). This principle says that if for two acts consequences are in some sense equally likely, then the acts are equivalent.

Corollary 4.2: In Statement ( $i$ ) of Theorem 3.1 additivity of the capacity can be added if in Statement (ii) P4 (cumulative dominance) is replaced by P4" (reduction) plus the restriction of $\mathrm{P} 4$ to two-consequence acts.

The above corollary can be regarded as a generalization of the result of Anscombe and Aumann (1963) and Fishburn (1967). Their structure is rich enough to satisfy P1-P3, P4", and P5-P7. The set-up of the above corollary is more general in exactly the same way that the set-up of Theorem 3.1 is more general than the result of Schmeidler (1989): The state space is not required to be a Cartesian product of ambiguous and unambiguous events. All that is needed is that the set of unambiguous events be rich enough. In the same way that Theorem 3.1 can be considered a unification of the results of Schmeidler (1989) and Gilboa (1987), the above corollary can be considered a unification of the results of Anscombe and Aumann (1963) and Savage (1954). The key feature in either case is that the events generated by a random device are incorporated within the state space. We think this is more natural than the two-stage approach of Anscombe and Aumann (1963). In the practice of decision analysis, objective probabilities of events $A^{u a}$ generated by a roulette wheel will typically be used as in Lemma A.1 in the Appendix to elicit "unknown" probabilities. This in no way requires a two-stage structure. While Theorem 3.1 was (apart from convex-rangedness) less general than Gilboa's result, the above corollary is a generalization of both Anscombe and Aumann's result and Savage's result. A generalization as indicated in Remark 3.3 can also be obtained for the above corollary.

An earlier result along these lines, within the classical additive set-up, is Bernardo, Ferrandiz, and Smith (1985). Corollary 4.2 is more general, mainly because, unlike Bernardo et al., we do not require a stochastic independence relation as a primitive, or existence of independent unambiguous events. 


\section{NONEQUIVALENCE OF ONE- AND TWO-STAGE APPROACHES}

Schmeidler made the novel contribution of showing that CEU is capable of permitting attitudes toward ambiguity that are disallowed by Savage's SEU. Schmeidler stated his axioms using the horserace-roulette wheel set-up of Anscombe and Aumann (1963). This is a two-stage set-up; i.e., in the first stage an event (e.g., the horse Secretariat winning) obtains and in the second stage the consequence is determined depending, for example, on a roulette wheel. In Schmeidler's model capacities are assigned to first-stage events. Further, the lotteries in the second stage are evaluated by the usual additive expected utility. An act assigns to each first-stage event a lottery, thus an expected utility value. The Choquet integral of these (with respect to the capacity over the first-stage events) gives the evaluation of the act. In our one-stage approach we embed the roulette wheel lotteries within Savage's formulation by enlarging the state space $S$. Our one-stage approach is complementary to the two-stage approach of Schmeidler as it provides additional flexibility in modeling decisions under uncertainty. This one-stage approach to CEU was introduced in Becker and Sarin (1989).

In the SEU theory, whether the one-stage or a two-stage approach is employed is purely a matter of taste or convenience in modeling. In the CEU framework, however, these two variations produce theoretically different results. We demonstrate this theoretical nonequivalence of one-stage and two-stage approaches through an example. Our analysis gives further evidence that multi-stage set-ups in nonexpected utility may cause complications. Gärdenfors and Sahlin (1983), Luce and Narens (1985), Luce (1988), Luce (1991), Luce (1992), Luce and Fishburn (1991), Segal (1987), and Segal (1990) focus on distinctions between one- and two-or-more-stage set-ups. Segal (1990) uses a two-stage set-up to describe an ambiguous event. Probabilities within each stage are assumed to be additive but they do not follow multiplicative rules between the two stages. Segal showed how dominance type axioms can provide nonexpected utility characterizations in the two-stage set-up (also see Wakker (1991b)).

EXAMPLE 5.1: This example is a small variation on one of the paradoxes of Ellsberg. The preferences used in the example below are consistent with those observed in the Ellsberg paradox. Further, the single-stage capacities are uniquely determined by the equivalent two-stage model of Schmeidler.

Suppose a biased coin and an unbiased coin will be tossed. The possible states of nature are $H^{b} H^{u b}, H^{b} T^{u b}, T^{b} H^{u b}, T^{b} T^{u b}$, where $H^{b} T^{u b}$ denotes the state where the biased coin lands heads up and the unbiased coin lands tails up, and so on. For simplicity assume that utility is known and that payment is in utility. It follows in Schmeidler's model that subjects consider a bet of 1 on $H^{u b 6}$ as well as a bet of 1 on $T^{u b}$ equivalent to $1 / 2$ for certain (given that payment is in utility). It has been observed that subjects will typically consider a bet of 1 on $H^{b}$ as well as a bet of 1 on $T^{b}$ less preferable. Let us assume the latter bets are equivalent to $\alpha$ for certain, for some number $\alpha<1 / 2$.

${ }^{6}$ Such a bet gives 1 if $H^{u b}$ obtains and 0 if $T^{u b}$ obtains. 
act

stage 1

stage 2

consequences

(utilities)
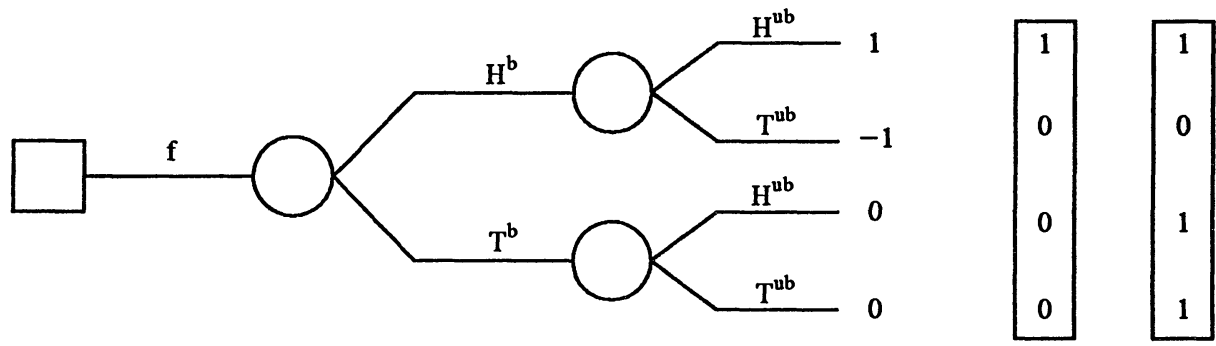

(a)

Figure 1a.-Two stage formulation of Example 7.
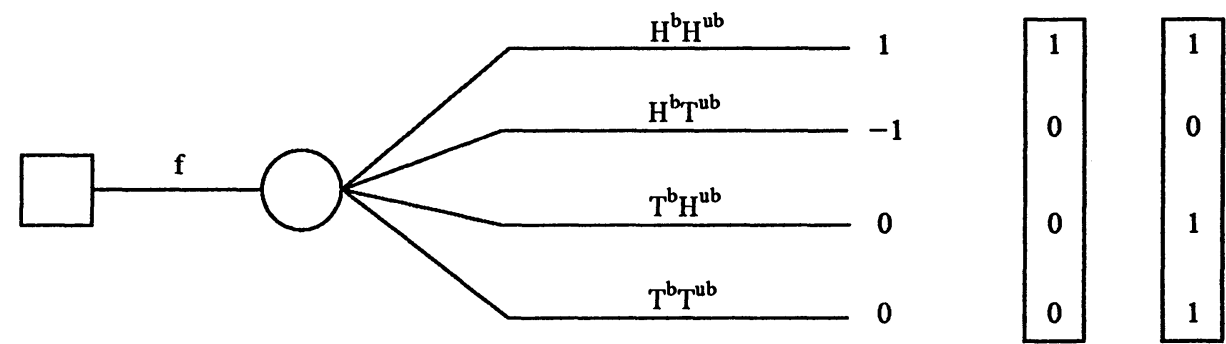

(b)

FIGURE 1b.--One stage formulation of Example 7.

In the two-stage set-up of Anscombe-Aumann and Schmeidler, decisions are formulated as shown in Figure 1a. For the act $f$ shown in Figure 1a, the two-stage approach yields CEU $(f)=0$, because the probability of $H^{u b}$ and $T^{u b}$ is $1 / 2$. Thus, $f$ is judged indifferent to a constant act $g$ with consequence 0 . Note that our assumption stated in the preceding paragraph implies that, with $v^{m}$ denoting the capacity in the two-stage approach, $v^{m}\left(H^{b}\right)=v^{m}\left(T^{b}\right)=\alpha$.

Now consider the one-stage formulation of the act in Figure 1a as depicted in Figure $1 \mathrm{~b}$. To evaluate $\operatorname{CEU}(f)$ in Figure $1 \mathrm{~b}$ we need the single-stage capacities, now denoted $v^{j}$ to distinguish from the capacities in the two-stage approach, $v^{j}\left(H^{b} H^{u b}\right)$ and $v^{j}\left(H^{b} H^{u b}, T^{b} H^{u b}, T^{b} T^{u b}\right)$. For consistency with the two-stage approach (see the boxed columns in Figures 1a and 1b), the first column in Schmeidler's two-stage approach is equivalent to $\alpha / 2$ and the second column to $\alpha \times 1+(1-\alpha) \times \frac{1}{2}=\frac{1}{2}+\frac{1}{2} \alpha$, so $v^{j}\left(H^{b} H^{u b}\right)=\alpha / 2$ and $v^{j}\left(H^{b} H^{u b}, T^{b} H^{u b}, T^{b} T^{u b}\right)=\frac{1}{2}+\frac{1}{2} \alpha$ must be chosen. Hence, in the one-stage approach, $\operatorname{CEU}(f)$ is $\alpha / 2+(1-(1 / 2+\alpha / 2))(-1)=\alpha-\frac{1}{2}<0$; it follows that $f \prec g(\equiv 0)$. Thus the one-stage approach and the two-stage approach yield different results, and are irreconcilable. They only agree in the additive case $\alpha=1 / 2$. 
In Sarin and Wakker (1990, Theorem 10) it is shown that the result of the above example holds in full generality. That is to say, only under expected utility can the one- and two-stage approach of CEU be equivalent. As soon as the capacity is nonadditive in Schmeidler's two-stage approach, the equivalent one-stage approach is not a CEU model.

\section{CONCLUSION}

Savage's SEU theory is widely accepted as a rational theory of decision making under uncertainty in economics and decision sciences. Unfortunately, however, people's choices violate the axioms of SEU theory in some welldefined situations. One such situation is when event probabilities are ambiguous. In this paper we have shown that a simple extension of SEU theory called Choquet expected utility (CEU) theory can be derived by assuming a natural cumulative dominance condition. CEU permits a subject to assign probabilities to events so that the probability of a union of two disjoint events is not necessarily the sum of the individual event probabilities. The violation of additivity may occur because a person's choice may be influenced by the degree of confidence or specificity about the event probabilities.

Schmeidler and Gilboa have also proposed axioms to derive the CEU representation. Building on their work, we have provided the simplest derivation of CEU presently available. Also, conditions have been given under which CEU reduces to SEU. It is also shown that unlike SEU theory, where a one-stage set-up of Savage or a two-stage set-up of Anscombe and Aumann yield identical results, the two-stage CEU formulation of Schmeidler cannot be reconciled with a one-stage formulation unless event probabilities are additive. In our opinion the one-stage set-up as used by Gilboa seems more appropriate in single-person decision theory. We hope that our work has clarified the distinction between CEU and SEU theories and that it will stimulate further research and additional explorations of CEU.

Anderson Grad. School of Management, University of California-Los Angeles, Los Angeles, CA 90024, U.S.A.

and

Dept. of Mathematical Psychology, University of Nijmegen, P.O. Box 9104, 6500 HE Nijmegen, The Netherlands

Manuscript receiced September, 1990; final revision received April, 1992.

APPENDEX: ProOFS

A1. Proof of Theorem 3.1, Remark 3.3, and Obsercation 3.4

For the implication (i) $\Rightarrow$ (ii) in Theorem 3.1, suppose (i) holds. Then P1 follows directly. P2 and P3 are standard results from, mainly, the usual additive expected utility theory. For Postulate P4, note that if $\left[f^{-1}(E) \geqslant g^{-1}(E)\right.$ for all cumulative consequence sets $\left.E\right]$, then by Lemma 2.1 the 
integrand in (1) is at least as large for $\phi=U \circ f$ as for $\phi=U \circ g$. So $f \geqslant g$, as P4 requires. P5 is direct from nonconstantness of $U$. For P6, let $f \in \mathscr{F}$ ua, $g \in \mathscr{F}, f \succ g$ (the case $f \prec g$ is similar) and $\alpha \in \mathscr{b}$. By boundedness of utility, there exists $\mu>0$ such that $\forall s \in S: U(f(s))-U(\alpha)<\mu$. Because $v$ is convex-ranged within $\mathscr{A}^{u a}$, we can take a partition $\left\{A_{1}, \ldots, A_{m}\right\}$ of $S$ such that $A_{j} \in \mathscr{A}^{u a}$ and $v\left(A_{j}\right)<(\operatorname{CEU}(f)-\operatorname{CEU}(g)) / \mu$ for all $j$.

For P7, let $f, g \in \mathscr{F}$, and let $A \in \mathscr{A}$ be a nonempty event ( $f$-convexity of $A$ will not be used). Then, with $U^{*}=U \circ f$ on $A^{c}$, and $U^{*}=\inf _{A} U \circ f$ (inf is real-valued by nonemptiness of $A$ and boundedness of $U$ ) on $A$, the premise in P7 implies

$$
\int U \circ f d v \geqslant \int U^{*} d v=\inf _{\mathrm{s} \in A}\left(\int U \circ f(s)_{A} f d v\right) \geqslant \operatorname{CEU}(g) .
$$

Next we suppose (ii) holds, and derive (i) and the uniqueness results, including Observation 3.4. It is immediate that Savage's postulates P1-P6 hold true on $\mathscr{F}$ ua. So we get an SEU representation on $\mathscr{F}$ s.ua, which denotes the set of step acts in $\mathscr{F} "$ ua. There exist a cardinal utility function $U: b \rightarrow \mathbb{R}$ and a unique additive probability measure $P$ on $\mathscr{A}^{\prime \prime a}$, such that expected utility represents preferences on $\mathscr{F}^{s, u a}$. We call $P(A)$ the "probability" of $A$. As follows from Savage (1954), $P$ is atomless and satisfies convex-rangedness. Obviously, $P$ will be the restriction of $v$ to $\mathscr{F} u a$.

Let us next extend the CEU representation as now established for all unambiguous step acts, to all step acts. First we define the capacity $v$. By P5 there are consequences $\zeta \succ \eta$, which are kept fixed throughout the proof.

\section{Lemma A.1: For each event $A$ there exists an $A^{u a} \in \mathscr{A}^{u a}$ such that $\zeta_{A} \eta \sim \zeta_{A^{u a}} \eta$.}

Proof: By P2, $\zeta_{S} \eta \geqslant \zeta_{A} \eta \geqslant \zeta_{\varnothing} \eta$. Suppose that in fact $\zeta_{S} \eta \succ \zeta_{A} \eta \succ \zeta_{\varnothing} \eta$ (otherwise we are done immediately), and that for event $B^{u a} \in \mathscr{A}^{u a}$ we have $\zeta_{A} \eta \succ \zeta_{B^{u a}} \eta$ (e.g., $B^{u a}=\varnothing$ ). This implies $P\left(\left(B^{u a}\right)^{c}\right)>0$. By P6, there exists a partition $C_{1}, \ldots, C_{n}$ of $S$, with all $C_{,} \in \mathscr{A}^{u a}$, such that $\zeta_{B^{\prime \prime a} \cup C_{j} \eta} \prec \zeta_{A} \eta$ for all $j$. There exists at least one $C_{j} \cap\left(B^{\prime \prime \prime}\right)^{c}$ with strictly positive probability. So there exists an event $B^{\prime \prime a^{\prime}}:=B^{\prime \prime a} \cup C_{j}$ with probability strictly greater than $B^{\prime \prime \prime}$, and such that still $\zeta_{A} \eta \succ \zeta_{B^{u a}} \eta$. So, using convex-rangedness, the set of probabilities of events $B^{\text {ua } a}$ as above must be of the form $\left[0, p^{-}\left[\right.\right.$for some $0<p^{-} \leqslant 1$.

Similarly, the set of probabilities of events $C^{u a} \in \mathscr{A}^{u a}$ such that $\zeta_{A} \eta \prec \zeta_{C^{u a}} \eta$, must be of the form ] $p^{+}, 1$ ] for some $0 \leqslant p^{+}<1$. The only possibility is $p^{-}=p^{+}$. By convex-rangedness there exists an event $A^{u a} \in \mathscr{A}^{u a}$ with probability $p^{-}$. Now $\zeta_{A} \eta \sim \zeta_{A^{u a}} \eta$ is the only possibility.

Q.E.D.

Thus, for every $A \in \mathscr{A}$, there exists an $A^{u a}$ that is equally likely. Because each possible choice of $A^{u a}$ has the same $P$ value, we can define $v: A \mapsto P\left(A^{u a}\right)$, extending $v$ from $\mathscr{A}^{u a}$ (where $v=P$ ) to the entire $\mathscr{A}$. For monotonicity with respect to set-inclusion, suppose that $A \supset B$. Then, by $\mathrm{P} 2$, $\zeta_{A} \eta \geqslant \zeta_{B} \eta$. From this $v(A) \geqslant v(B)$ follows, and $v$ is a capacity.

To establish the CEU representation for all step acts, we construct for each ambiguous step act an unambiguous one "with the same cumulative distribution." That is, for the ambiguous and the unambiguous acts the events of obtaining a consequence at least as good as $\alpha$ are equally likely, for each consequence $\alpha$. For step acts this is not only necessary, but also sufficient, to have all cumulative consequence sets equally likely under the two acts. First we extend Lemma A.1. The proof of the extension is completely similar, with $\mu, \nu$ in the place of $\zeta, \eta$, further $f$ in the place of $\zeta_{A} \eta$, and $\mu \geqslant f \geqslant \nu$ implied by $\mathrm{P} 4$.

Lemma A.2: For each act $f$ for which there exist consequences $\mu, \nu$ such that $[\forall s \in S: \mu \geqslant f(s) \geqslant \nu]$, there exists an $A^{u a} \in \mathscr{A}^{u a}$ such that $\mu_{A^{u a}} \nu \sim f$.

Obviously, by the SEU representation as already established, $\zeta_{A^{m a}} \eta \sim \zeta_{B^{\prime \prime \prime}} \eta$ for each unambiguous event $B^{u a}$ equally likely as $A^{u a}$. By convex-rangedness of $P$, and Lemma A.1, for each partition $A_{1}, \ldots, A_{m}$ of $S$ we can find an unambiguous partition $B_{1}, \ldots, B_{m}$ of $S$ such that $A_{1} \cup \cdots \cup A_{j}$ is equally likely as $B_{1} \cup \cdots \cup B_{j}$, for each $j$. To do so, first we find an unambiguous $B_{1}^{\prime} \sim A_{1}$, and set $B_{1}:=B_{1}^{\prime}$. Next we find an unambiguous $B_{2}^{\prime} \sim A_{1} \cup A_{2}$. By convex-rangedness of $P$, we can find an unambiguous $B_{2}$ with $B_{2} \cap B_{1}=\varnothing$ such that $P\left(B_{1} \cup B_{2}\right)=P\left(B_{2}^{\prime}\right)$, so that $B_{1} \cup B_{2} \sim A_{1} \cup A_{2}$, and so on. The next paragraph is the central part of the proof, and is simple. The other parts of the proof are all standard after Savage (1954), using Gilboa's (1987) P7.

Let $\alpha_{A_{1}}^{1} \ldots \alpha_{A_{m}}^{m}$ be an arbitrary step act, with $\alpha^{1} \succcurlyeq \ldots \succcurlyeq \alpha^{m}$. We take an unambiguous partition $\left\{B_{1}, \ldots, B_{m}\right\}$ as described above. The unambiguous act $\alpha_{B_{1}}^{1} \ldots \alpha_{B_{m}}^{m}$, by two-fold application of 
P4 (once with $\succcurlyeq$, once with $\preccurlyeq$ ), is equivalent to the ambiguous act. Its SEU value can, similarly to the Choquet integral, be written as $P\left(B_{1}\right) U\left(\alpha^{1}\right)+\left[P\left(B_{1} \cup B_{2}\right)-P\left(B_{1}\right)\right] U\left(\alpha^{2}\right)+\cdots+[1-$ $\left.P\left(B_{1} \cup \ldots \cup B_{m-1}\right)\right] U\left(\alpha^{m}\right)$. This shows that it is identical to the CEU value of the ambiguous act. So indeed CEU represents preferences between all step acts.

The extension of the CEU representation to non-step acts is mainly by $\mathrm{P} 7$, and is similar to Gilboa (1987). Note that this in particular establishes the expected utility representation on the entire set $\mathscr{F}^{u a}$. Contrary to Gilboa (1987), our capacity need not be convex-ranged. We can however follow the reasoning of his Subsection 4.3 with only unambiguous step acts $\bar{f}, \bar{g}$. Convexrangedness is used there for the existence of $\bar{g}$, while convex-rangedness of $P$ suffices for that. In the proof of his Theorem 4.3.4, in Statement (i), the act $\bar{f}$ can always be chosen unambiguous, by Lemma A.2. Let us also mention that one cannot restrict P7 to $\mathscr{F}^{u a}$. This would be possible if for each ambiguous act there would exist an unambiguous act with the same cumulative distribution. This however is not the case in general. For example if $P$ is countably additive, then it cannot generate strictly finitely additive distributions; e.g., with $b=\mathbb{R}$, it does not generate cumulative distribution functions that are not continuous from the right. Also it is possible that for instance $U(b)=[0,1[, P$ is countably additive, and there exists a positive $\varepsilon$ such that under an ambiguous act $f$ each cumulative event $\{s \in S: f(s) \geqslant \alpha\}(0 \leqslant \alpha<1)$ has capacity at least $\varepsilon$.

The utility functions must be bounded, as follows from the representation on $\mathscr{F}$ ua. This is shown in Fishburn (1970, Section 14.1), and the second 1972 edition of Savage (1954, footnote on p. 80). Finally we establish the uniqueness results. By the standard results of Savage (1954) we get cardinality of $U$, and uniqueness of the restriction $P$ of $v$ to $\mathscr{A}^{u a}$. The extension of $v$ to $\mathscr{A} \backslash \mathscr{A}^{\text {ua }}$ shows that $v$ is uniquely determined.

Next let us suppose that $v$ is allowed to be nonadditive on $\mathscr{F}^{\text {ua }}$, as studied in Observation 3.4. Let us at first also suppose that there are three or more nonequivalent consequences. Then the representation, if restricted to $\mathscr{F}^{\text {ua }}$, satisfies all conditions in Gilboa (1987); hence by his uniqueness results the restriction of $v$ to $\mathscr{F}^{\text {ua }}$ is unique, so additive. The uniqueness of $v$ follows in the same way as above. Let us finally consider the case where there are exactly two equivalence classes of consequences, with say $\zeta \succ \eta$. Any $U^{\prime}$ instead of $U$ in a CEU representation is constant on equivalence classes of consequences and satisfies $U(\zeta)>U(\eta)$. So $U^{\prime}$ is a strictly increasing transform of $U$, and obviously is bounded. Given the two-valued range, $U$ is cardinal. Because any $v^{\prime}$ in a CEU representation has to represent the same ordering over events as $v, v^{\prime}$ must be a strictly increasing transform of $v$. Conversely, any such $v^{\prime}$ will do. Thus it is possible to choose $v$ such that it is not convex-ranged and not additive on $\mathscr{A}^{u a}$. It can however always be chosen such that it is convex-ranged and additive on $\mathscr{A}^{\text {ua }}$.

For the proof of Remark 3.3, note that all constructions in the proof of the implication (ii) $\Rightarrow$ (i) of Theorem 3.1 (including the extension to nonsimple acts, following Gilboa (1987)) remain possible under the conditions of Remark 3.3.

Our result has not established convex-rangedness of the capacity $v$. That can be characterized by addition of one condition, Gilboa's P6*. We propose to rename this as "solvability." Solvability is satisfied if for all acts $f, g$, consequences $\alpha \succ \beta$, and events $A$, if $\alpha_{A} f \succ g \succ \beta_{A} f$, and $\alpha_{A} f, \beta_{A} f$ "comonotonic" $\left(\forall s \in A^{c}: f(s) \succcurlyeq \alpha\right.$ or $\left.f(s) \preccurlyeq \beta\right)$, there exists an event $B \subset A$ such that $\alpha_{B} \beta_{A \backslash B} f_{A^{c}} \sim$ $g$. That solvability, even if restricted to two-consequence acts, is sufficient for convex-rangedness of $v$, follows mainly from convex-rangedness of $P$, which gives all desired "intermediate" $g$. Necessity is straightforward.

Proposition A.3: Suppose Statement ( $i$ ) in Theorem 3.1 holds. Then $v$ is concex-ranged if and only if $\succcurlyeq$ satisfies solvability.

For the case of three or more equivalence classes of consequences, a more general derivation, without use of $\mathscr{F}^{u a}$, is given in Gilboa (1987). If there are exactly two equivalence classes of consequences and $v$ is not required to be additive on $\mathscr{A}^{u a}$, then, by Observation $3.4, v$ need not be convex-ranged, even if solvability is satisfied.

The following example shows why we used cumulative consequence sets, instead of less general sets of the form $\{\beta \in \mathscr{b}: \beta \geqslant \alpha\}$ for $\alpha \in \mathscr{b}$, in the definition P4 of cumulative dominance, and its derivatives $\mathrm{P}^{\prime}$ and $\mathrm{P}^{\prime \prime}$. Note that the distinction is relevant only for nonstep acts, and that we could have restricted $\mathrm{P} 4, \mathrm{P}^{\prime}, \mathrm{P}^{\prime \prime}$ to step acts. In that case we could have used the less general sets as mentioned above.

ExAmple A.4: Suppose the special case of Statement (i) in Theorem 3.1 holds where in fact all of Savage's axioms are satisfied. So $v$ is an additive probability measure, that we denote by $P$. Let 
$\mathscr{b}=\{-1 / j: j \in \mathbb{N}\} \cup\{1+1 / j: j \in \mathbb{N}\}$, and let $U$ be the identity. Let $\left\{A_{j}\right\}_{j=1}^{\infty} \cup\left\{B_{j}\right\}_{j=1}^{\infty}$ be a partition of $S,\left\{A_{j}^{\prime}\right\}_{j=1}^{\infty} \cup\left\{B_{j}^{\prime}\right\}$ another partition of $S . A:=\cup_{j=1}^{\infty} A_{j}, B, A^{\prime}, B^{\prime}$ are defined similarly. Suppose that $P\left(A_{j}\right)=P\left(A_{j}^{\prime}\right), \quad P\left(B_{j}\right)=P\left(B_{j}^{\prime}\right)$ for all $j$. Further suppose that $P(A)>P\left(A^{\prime}\right)$. Such cases can be constructed if $P$ is not set-continuous, i.e., not countably additive. Let $f$ assign $1+1 / j$ to each $A_{j}$, and $-1 / j$ to each $B_{j}$. Similarly $f^{\prime}$ assigns $1+1 / j$ to each $A_{\jmath}^{\prime}$, and $-1 / j$ to each $B_{j}^{\prime}$. For each consequence $1+1 / j$ we have $P(f(s) \geqslant 1+1 / j)=\sum_{i=1}^{J} P\left(A_{j}\right)=\sum_{i=1}^{j} P\left(A_{j}^{\prime}\right)=$ $P\left(f^{\prime}(s) \geqslant 1+1 / j\right)$. For each consequence $-1 / j$ we have $P(f(s) \geqslant-1 / j)=1-P(f(s) \prec-1 / j)=$ $1-\sum_{i=1}^{j-1} P\left(B_{i}\right)=1-\sum_{i=1}^{j-1} P\left(B_{i}^{\prime}\right)=P\left(f^{\prime}(s) \geqslant-1 / j\right)$. So for each $\alpha \in \mathscr{b}:\{s \in S: f(s) \geqslant \alpha\} \sim$ $\left\{s \in S: f^{\prime}(s) \geqslant \alpha\right\}$. However, for $0 \leqslant \mu \leqslant 1, P(f(s) \geqslant \mu)=P(A)>P\left(A^{\prime}\right)=P\left(f^{\prime}(s) \geqslant \mu\right)$. By Formula (1), $\operatorname{CEU}(f)-\operatorname{CEU}\left(f^{\prime}\right)=1 \times\left(P(A)-P\left(A^{\prime}\right)\right)>0$. So $f \succ f^{\prime}$. Only for cumulative consequence sets $E:=\left[\mu, \infty\left[\right.\right.$ with $\mu$ as above we do not have $f^{-1}(E) \geqslant f^{-1}(E)$.

\section{A2. Proof of Proposition 4.1.}

The implications (i) $\Rightarrow$ (ii) and (i) $\Rightarrow$ (iv) are direct. The implication (i) $\Rightarrow$ (iii) follows from convex-rangedness of $P$. Next we prove that Statement (i) is implied by each of the other statements. (ii) $\Rightarrow$ (i) is direct. (iii) $\Rightarrow$ (ii) follows from taking $A$ and $A^{\prime}$ as union of $A$,'s, taking $B^{\prime \prime a}$ and $B^{\prime \prime \prime \prime}$ as union of corresponding $B_{J}^{u a \prime}$ 's, and from the equivalences (with $\zeta \succ \eta$ ) $\zeta_{A} \eta \sim \zeta_{B^{\prime \prime \prime}} \eta$, $\zeta_{A^{\prime}} \eta \sim \zeta_{B^{\text {tua }}} \eta, \zeta_{A \cup A^{\prime}} \eta \sim \zeta_{B^{\text {utu }} \cup B^{\text {um }}, \eta}$. Finally, suppose (iv) holds. Similarly to the reasoning below Lemma A.2, we can show the existence of an unambiguous partition $\left\{B_{1}^{\prime \prime a}, \ldots, B_{m}^{\prime \prime \prime}\right\}$ such that $A_{1} \cup \cdots \cup A_{j} \sim B_{1}^{u a} \cup \cdots \cup B_{j}^{u a}$ for all $j$. For any $A$ that is a union of $A_{j}$ 's-different-from- $A_{1}$, and $B^{\prime \prime a}$ a union of corresponding $B_{j}$ 's, we have, by (2), $\zeta_{A \cup A_{1}} \eta \sim \zeta_{B^{u a}} \cup B_{1}^{\prime \prime \prime} \eta$ and $\zeta_{A} \eta \sim \zeta_{B^{u a}} \eta$. Taking differences and dividing by the positive $U(\zeta)-U(\eta)$ we get $v\left(A \cup A_{1}\right)-v(A)=P\left(B^{\prime \prime a} \cup B_{1}^{\prime \prime a}\right)-$ $P\left(B^{u a}\right)=P\left(B_{1}^{\prime \prime a}\right)$. So the "decision weight" that $A_{1}$ contributes to each union of the other $A$, 's, is independent of those other $A_{j}$ 's. The same holds for each $A_{i}$. Hence the capacity of a union of different $A_{j}$ 's is the sum of the separate capacities: $v$ is additive on the partition.

\section{REFERENCES}

Anscombe, F. J., And R. J. Aumann (1963): “A Definition of Subjective Probability," Annals of Mathematical Statistics, 34, 199-205.

Becker, J. L., AND R. K. SARIN (1989): "Economics of Ambiguity,” Duke University, Fuqua School of Business, Durham, NC, USA.

Bernardo, J. M., J. R. Ferrandiz, and A. F. M. Smith (1985): "The Foundations of Decision Theory: An Intuitive, Operational Approach with Mathematical Extensions," Theory and Decision, 19, 127-150.

Choouet, G. (1953-1954): “Theory of Capacities," Annales de l'Institut Fourier, 5 (Grenoble), 131-295.

DE Finetrı, B. (1931): "Sul Significato Soggettivo della Probabilità," Fundamenta Mathematicae, 17, $298-329$.

(1937): "La Prévision: Ses Lois Logiques, ses Sources Subjectives," Annales de l'Institut Henri Poincaré, 7, 1-68. Translated into English by H. E. Kyburg, "Foresight: Its Logical Laws, its Subjective Sources," in Studies in Subjectice Probability, ed. by H. E. Kyburg and H. E. Smokler. New York: Wiley, 1964, 53-118; 2nd edition, New York: Krieger, 1980.

Ellsberg, D. (1961): "Risk, Ambiguity and the Savage Axioms," Quarterly Journal of Economics, $75,643-669$.

Fishburn P. C. (1967): "Preference-Based Definitions of Subjective Probability," Annals of Mathematical Statistics, 38, 1605-1617.

(1970): Utility Theory for Decision Making. New York: Wiley.

(1982): The Foundations of Expected Utility. Dordrecht: Reidel.

(1988): Nonlinear Preference and Utility Theory. Baltimore: Johns Hopkins University Press.

(1991): "On the Theory of Ambiguity," International Journal of Information and Management Science, 2, 1-16.

Gärdenfors, P., AND N.-E. SAhlin (1983): "Decision Making with Unreliable Probabilities," British Journal of Mathematical and Statistical Psychology, 36, 240-251.

GilboA, I. (1987): "Expected Utility with Purely Subjective Non-Additive Probabilities," Journal of Mathematical Economics, 16, 65-88.

(1989): "Duality in Non-Additive Expected Utility Theory," in Choice under Uncertainty, Annals of Operations Research, ed. by P. C. Fishburn and I. H. LaValle. Basel: J. C. Baltzer AG, 405-414. 
Keynes, J. M. (1921): A Treatise on Probability. London: McMillan. Second edition, 1948.

KNight, F. H. (1921): Risk, Uncertainty, and Profit. New York: Houghton Mifflin.

LuCE, R. D. (1988): “Rank-Dependent, Subjective Expected-Utility Representations," Journal of Risk and Uncertainty, 1, 305-332.

(1991): "Rank- and-Sign Dependent Linear Utility Models for Binary Gambles," Journal of Economic Theory, 53, 75-100.

- (1992): "Where Does Subjective Expected Utility Fail Descriptively?" Journal of Risk and Uncertainty, 4, 5-27.

Luce, R. D., And P. C. Fishburn (1991): "Rank- and-Sign Dependent Linear Utility Models for Finite First-Order Gambles," Journal of Risk and Uncertainty, 4, 29-59.

LucE, R. D., AND L. Narens (1985): "Classification of Concatenation Measurement Structures According to Scale Type,” Journal of Mathematical Psychology, 29, 1-72.

Machina, M. J. (1982): “'Expected Utility' Analysis without the Independence Axiom,” Econometrica, 50, 277-323.

Machina, M. J., And D. J. Schmeidler (1990): “A More Robust Definition of Subjective Probability," Dept. of Economics, University of California, San Diego.

NaKamura, Y. (1990): "Subjective Expected Utility with Non-Additive Probabilities on Finite State Spaces," Journal of Economic Theory, 51, 346-366.

(1992): "Multi-Symmetric Structures and Non-Expected Utility," Journal of Mathematical Psychology, forthcoming.

RAMSEY, F. P. (1931): "Truth and Probability," in The Foundations of Mathematics and other Logical Essays. London: Routledge and Kegan Paul, 156-198. Reprinted in Studies in Subjectice Probability, ed. by H. E. Kyburg and H. E. Smokler. New York: Wiley, 1964, 61-92.

SARIN, R., AND P. P. WAKKER (1990): "Incorporating Attitudes towards Ambiguity in Savage's Set-up," University of California, Los Angeles, CA.

Savage, L. J. (1954): The Foundations of Statistics. New York: Wiley. Second Edition, New York: Dover, 1972.

Schmeidler, D. (1989): “Subjective Probability and Expected Utility without Additivity," Econometrica, 57, 571-587.

Segal, U. (1987): "The Ellsberg Paradox and Risk Aversion: An Anticipated Utility Approach," International Economic Review, 28, 175-202.

(1990): “Two-Stage Lotteries without the Reduction Axiom," Econometrica, 58, 349-377.

Wakker, P. P. (1989a): “Continuous Subjective Expected Utility with Nonadditive Probabilities," Journal of Mathematical Economics, 18, 1-27.

- (1989b): Additive Representations of Preferences, A New Foundation of Decision Analysis. Dordrecht: Kluwer.

- (1990a): "Unbounded Utility for Savage's 'Foundations of Statistics', and Other Models," Mathematics of Operations Research, forthcoming.

(1990b): "Under Stochastic Dominance Choquet-Expected Utility and Anticipated Utility are Identical," Theory and Decision, 29, 119-132. Approach," "Journal of Mathematical Psychology, 35, 501-531.

and Uncertainty, forthcoming.

YAARI, M. E. (1987): “The Dual Theory of Choice under Risk," Econometrica, 55, 95-115. 